Original Research

\title{
Grip Strength Measurement in Baseball Pitchers: A Clinical Examination to Indicate Stride Length Inefficiency
}

\author{
Ryan Crotin ${ }^{1}$, Dan Ramsey ${ }^{2}$ \\ ${ }^{1}$ ArmCare.com; Department of Exercise Science, School of Public Health and Health Professions, University at Buffalo; Sports Performance Research \\ Institute New Zealand, Auckland University of Technology; Human Performance Laboratories, Department of Kinesiology, Louisiana Tech University, ${ }^{2}$ \\ Department of Health Professions Education, School of Health Professions, D'Youville College \\ Keywords: pitching, forearm, dynamometry, key terms: lower body \\ https://doi.org/10.26603/001c.28086
}

\section{International Journal of Sports Physical Therapy}

Vol. 16, Issue 5, 2021

\section{Background}

Ulnar collateral ligament injuries are rampant in the sport of baseball where kinetic chain impacts, stemming from misappropriation of stride length or changes that occur in competition due to fatigue, have not been evaluated for dynamic elbow stability effects.

\section{Hypothesis/Purpose}

To examine the relationship between clinical measures of grip strength and altered stride length in baseball pitchers. It is believed that shorter stride lengths would reduce grip strength in baseball pitchers.

\section{Study Design}

Crossover Study Design

\section{Methods}

A total of 19 uninjured pitchers ( 15 collegiate and 4 high school) (age $18.63 \pm 1.67$ years, height $1.84 \pm 0.054 \mathrm{~m}$, mass $82.14 \pm 0.054 \mathrm{~kg}$ ) threw two simulated 80-pitch games at $\pm 25 \%$ of their desired stride length recorded by motion capture with two force plates and a radar gun to track each throw. A handheld grip dynamometer was used to record the mean change in grip strength after games from baseline measures. Pairwise comparisons at baseline and post-game denoted grip strength changes and dominant grip strength offsets for stride length conditions.

\section{Results}

Subjects with shorter stride lengths revealed a significant decline in grip strength in the dominant arm from baseline (pre-game; $45.1 \mathrm{~kg}$ vs. post-game; $43.2 \mathrm{~kg}, \mathrm{p}=0.017, \mathrm{ES}=0.28$ ), however all other tests involving dominant grip strength changes and offset analyses were not statistically different for under-stride and over-stride length conditions.

\section{Conclusions}

Clinical evaluation of grip strength has the potential to identify altered lower body mechanics and may be considered as a safe and effective monitoring strategy to integrate with motion capture in determining optimal stride lengths for baseball pitchers.

\section{Level of Evidence}

Level 3

\footnotetext{
a Corresponding author:

Vice President

ArmCare.com

255 5th Ave., Suite 2

Indialantic, FL 32903

Email: RLCrotin@gmail.com

Phone: (716) 998-9551
} 


\section{INTRODUCTION}

Elbow valgus torque is the primary mechanism responsible for ulnar collateral ligament (UCL) failure where dynamic stability offered by elbow musculature is critical to stress shielding of the UCL. ${ }^{1,2}$ Cadaveric research has shown the biceps, triceps and brachialis provide medial elbow stability at all degrees of forearm rotation. ${ }^{1}$ In addition to large, biarticular muscles, the flexor pronator mass appears to provide significant stability despite a deficient, anterior UCL bundle in a supinated wrist position, which is representative of the forearm position near peak valgus loading as the throwing arm lays back in the later stages of the cocking phase. ${ }^{1,3}$

Optical motion capture provides further context into biomechanical limitations posed by cadaveric loading simulations. However, motion capture presents a challenge as kinetics are expressed as net joint moments combining osseous, connective tissue and muscular contributions to the varus moment and does not identify individual muscles' force applications. Musculoskeletal modeling from threedimensional pitching analyses can elucidate the roles of specific, medial elbow, dynamic stabilizers that can be trained to apply greater varus torque to resist opening of the medial elbow. ${ }^{4}$ Pitching fatigue is the greatest contributor to injury, where electromyography-based modeling has shown how neural activation of forearm musculature is affected by workload and reinforces the need to study grip strength loss as a measure of functional forearm fatigue (FFF) of the flexor pronator mass. ${ }^{5,6}$

Pitching workload fatigue has been examined through functional strength changes, yet muscular weakness impacting kinematics and kinetics are unknown ${ }^{7,8}$. Similarly, biomechanical changes have been reported in pitching fatigue studies, yet the movement adaptations described cannot be considered compensations given velocity depreciated and was not maintained with increasing workload. ${ }^{9-11}$

In other work, stride length has been shown to vary with increased pitch counts that may be accompanied by changes in momentum, timing, physiology and ground reaction force. ${ }^{12-16}$ Compensation to maintain velocity through a reduction in stride length entailed increased total body angular momentum as a result of reduced ground reaction force in the anterior-posterior direction, altering transfers from trunk to throwing arm. ${ }^{14}$ Related to this paradigm, Kibler ${ }^{17}$ mentioned that altered transfer of ground reaction force from the lower body can reduce kinetic energy of the hip and trunk and thereby increase rotational velocity at the shoulder. Increased throwing arm velocity as a result of lower body force deficits has the potential to increase dynamic stability effort for the forearm musculature making the flexor pronator mass more fatigable. ${ }^{17}$ Altered lower body function and its impact on forearm strength has not been extensively studied, yet has been shown to impact blood and salivary biomarkers, as well as visual analog scores related to fatigue. ${ }^{13}$ Therefore, this study is novel as it is aimed to model variation in stride length and its impact on FFF to illustrate the importance of clinical evaluation of grip strength in practice and competitive environments to infer less than optimal kinetic chain interactions. The purpose of the study was to examine the relationship between clinical measures of grip strength and altered stride length in baseball pitchers. We hypothesized that FFF of the medial elbow stabilizers will increase with shorter stride lengths (a surrogate for reduced ground reaction force production) to signify less than optimal stride length or pitching fatigue that alters lower body functioning.

\section{METHODS}

A cohort of 20 healthy and skilled competitive pitchers were recruited from collegiate and high school seasonal travel programs from across western New York. One pitcher withdrew owing to conflicts with collegiate baseball obligations. Of the remaining 19 participants, 15 were right-handed and four were left handed (age $18.63 \pm 1.67$ years, height 1.84 $\pm 0.054 \mathrm{~m}$, mass $82.14 \pm 0.054 \mathrm{~kg}) .{ }^{14,16,18}$ None of the athletes had experienced a throwing arm injury that required surgery. All had at least five seasons of competitive experience, were injury free within two years prior to participation, or had fully recovered from all previous injuries and were medically cleared. Prior to participating informed consent was approved by the University at Buffalo's Children and Youth Institutional Review Board, and subject signatures were obtained or parental consent granted for minors.

Upon arrival to the laboratory, intake procedures were initiated which included informed consent review, recording height and weight measurements and collecting baseline grip strength measurements as described in other work. ${ }^{19}$ Two calcaneal markers were affixed to the athlete's athletic shoes to be able to discern stride length from threedimensional (3D) motion capture data (Vicon Motion Systems, Denver, Colorado) and determine simulated game conditions. Stride lengths were determined as the horizontal distance along the global leading axis (horizontal distance from the first or third base perspective) between the drive foot calcaneus at peak knee height to the stride foot calcaneus at stride foot contact.

The instant of stride foot contact was identified by an unpublished pilot study involving force plates, undertaken by the principal investigator, that involved visual inspection of stride foot contact and its corresponding ground reaction force threshold. Pilot research indicated that a registered vertical ground reaction force that exceeded $5 \%$ body weight signified the minimum threshold to be considered foot contact. Also in the pilot study, the throwing hand and baseball were tracked with markers to indicate peak throwing hand velocities at the point of ball release.

Two force plates (Kistler Corp, Amherst, New York) were aligned in series with the area over the force plates marked to target drive foot and stride foot placement for each stride length intervention. Two subjects were taller than $1.93 \mathrm{~m}$ and had longer stride lengths than what the arrangement could accommodate. As a result, the starting point was moved back for these athletes to ensure the stride foot could target the second force platform. These athletes alternated their drive foot placement on and off the first force plate, so that propulsive ground reaction recordings could be captured for every other pitch, and thus both drive leg and stride leg ground reaction force could be recorded (Figure 1).

A randomized cross-over design was used where pitchers 
threw from flat ground in two 80-pitch simulated games on separate days with 72 hours rest between conditions before performing the other condition; 1 ) at $25 \%$ increased stride (OS), and 2) at $25 \%$ reduced stride (US) from desired stride length (DSL). The $\pm 25 \%$ change from desired stride length, measured in meters, was determined a-priori and was designed to challenge throwing mechanics. The raw stride length difference equated to a $24 \%$ difference in normalized stride length (\% body height) between conditions and fell between $50-80 \%$ body height (as measured during the warm-up), which is representative of collegiate and professional pitchers. ${ }^{10,20,21}$ The investigators used 50\% and $80 \%$ body height stride length as limits in the stride length spectrum to attempt to identify forearm grip strength changes that could be seen between extremes. Previous authors have reported stride length measures for the respective overstride and under-stride conditions in this study $(1.40 \pm 0.15$ and $0.95 \pm 0.14 \mathrm{~m})$ and normalized stride lengths $(0.76 \pm$ 0.07 and $0.52 \pm 0.08$ \%body-height). ${ }^{13-16}$ Pitchers adjusted throwing mechanics from the mean desired stride length $(1.24 \pm 0.17 \mathrm{~m}$ and $0.67 \pm 0.09 \%$ body-height $) .13$

Prior to simulated games, athletes underwent a standardized warm-up and were given time to prepare themselves with light throwing before initiating 25-30 warm-up pitches prior to simulated games that were recorded by motion capture. The last five warm-up pitches were thrown at $100 \%$ effort at the desired stride length. The two warm-up pitches with the highest velocities were averaged and desired stride length was determined from the kinematic data. At the end of warm-up throwing, ten pitches were thrown for each OS and US game condition to acclimatize pitchers to each simulated game condition.

None of the deliveries were side arm and, therefore, twenty over the top throws at $100 \%$ effort were completed per inning with a ratio of three fastballs to one changeup. The change-up was integrated into the simulated game to offload the throwing arm from delivering only fastballs. This workload allocation was determined from previous work examining short season, minor league baseball pitchers and conservatively estimated for high school, junior college and Division I baseball pitchers. ${ }^{22}$ With the use of a stopwatch, approximately 15 seconds of rest was allocated between pitches with a nine minute rest prescribed to simulate between inning rest periods. Five warm-up throws were allocated before each inning. Testing ceased after the $80^{\text {th }}$ delivery, with approximately 130-140 throws having been accumulated per testing condition. ${ }^{14,16,18}$ Ball velocities of all pitches thrown were tracked with a professional radar gun (Jugs Sports, Tualatin, OR).

Standard motion capture techniques involving eight cameras (VICON MX20 cameras, sampling at $240 \mathrm{~Hz}$ with Kistler force plates at $960 \mathrm{~Hz}$ ) and Visual 3D (C-Motion, Germantown, MD) software were used to record and analyze stride length measurements. Trials were eliminated from analysis when the athlete did not target the force platform for the over or under-stride length condition. To account for the potential of lower body fatigue, an ensemble average of the two highest velocity pitches in the first inning and last inning (four pitches total), were used to calculate in-game stride length measurements.

Grip strength was used to evaluate FFF by measuring

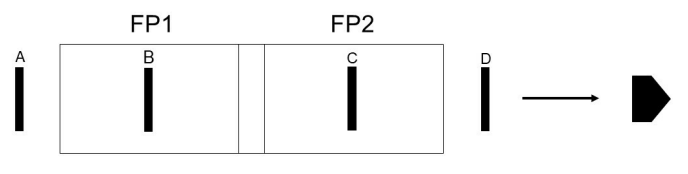

\section{Figure 1: Force plate configuration and target for pitching simulated games.}

\begin{abstract}
Force plates aligned in series. Arrow denotes the direction of the throw toward home plate.

FP1; Force plate 1 for the drive foot placement, FP2; Force plate 2 for the stride foot placement, A; Alternate drive foot target for pitchers with elongated stride lengths beyond constraints of the force plate mounting brackets, B; Drive foot target on force plate 1, C; Stride foot target on force plate 2, D; Alternate stride foot target for pitchers with elongated stride lengths beyond constraints of the force plate mounting brackets. Only pitchers with elongated stride lengths had to alternate their drive foot position with each pitch moving back and forth between $A$ and $B$, so that both drive and stride leg ground reaction forces could be captured.
\end{abstract}

force in kilograms on a calibrated, Jamar hydraulic hand dynamometer (Lafayette Instrument, Lafayette, IN). The baseline grip assessment was performed prior to the first simulated game and then prior to the second simulated game after 72 hours of rest to provide a pre-pitching baseline. Grip strength was then recorded immediately after both game conditions. Grip strength procedures were adapted from the work of Kobesova et al. ${ }^{19}$ Athletes sat in a chair with their feet contacting the ground flat-footed, hips and knees flexed at 90 degrees and the testing arm flexed 90 degrees at the elbow that was confirmed by goniometric measurement while maintaining a 0 degree pronation-supination wrist position. Athletes were required to squeeze the hand dynamometer maximally for 3 seconds, with loud verbal encouragement provided by the investigator. A stopwatch indicated 30 seconds between trials to provide recovery for each subsequent trial. An average grip strength over three trials was used in statistical analysis for both the dominant and non-dominant flexor pronator mass musculature. A dominant arm strength offset was calculated for both baseline and post-pitching by subtracting the average non-dominant grip strength from the average dominant grip strength, where a positive value indicated more dominant arm grip strength, while a negative result indicated stronger grip strength for the non-dominant arm.

Statistical analyses were performed using the 2016 version of Excel (Redmond, WA, USA). Paired t-tests compared pre- and post-game grip strength for both the dominant and non-dominant arms for each condition to examine FFF. Pairwise t-tests were also used to determine differences between conditions at baseline and post-game for dominant arm grip strength offsets. Statistical significance, determined a priori, was set at $p \leqslant 0.05$ for all statistical tests. Cohen's d effect size (ES) was calculated for paired t-tests that met significance using a pooled standard deviation and were interpreted as trivial (0-0.19), small (0.20-0.49), 
Table 1. Mean Pre and Post-Game Grip Strength Measures for Stride Length Conditions

\begin{tabular}{|l|c|c|}
\hline Measurement & Over-Stride & Under-Stride \\
\hline Pre-Game Dominant Grip Strength (kg) & 44.6 & $45.1^{*}$ \\
\hline Pre-Game Non-Dominant Grip Strength (kg) & 43.4 & 44.9 \\
\hline Post-Game Dominant Grip Strength (kg) & 43.4 & $43.2^{*}$ \\
\hline Post-Game Non-Dominant Grip Strength (kg) & 43.8 & 44.0 \\
\hline Baseline Dominant Arm Grip Strength Offset (kg) & 1.24 & 0.30 \\
\hline Post-Game Dominant Arm Grip Strength Offset (kg) & -0.41 & -0.82 \\
\hline
\end{tabular}

* Indicates that grip strength was reduced post-game versus baseline for the under-stride game condition ( $p=0.017, d=0.28$.

medium (0.50-0.79) and large (>0.80) effects. Results related to ground reaction force and ball velocity are reported in other work that indicated greater shear ground reaction forces in propulsion and braking with longer strides, yet no changes in ball velocity between stride length conditions. ${ }^{16,23}$

\section{RESULTS}

Following an 80-pitch simulated game, significant differences in FFF were not found between baseline and postpitching measures in the over-stride condition, nor differences in dominant grip strength offsets. In contrast, under-stride pitching revealed a significant decline in strength in the dominant arm from baseline; however, all other tests involving dominant grip strength offset analyses did not yield statistically significant differences (Table 1). All subject-specific data for grip strength for the dominant and non-dominant arm can be seen in Table 2. Potentiation effects, indicated by increased post-game grip strength for the dominant arm by $1 \mathrm{~kg}$ or greater in force, were seen in some subjects for both conditions. Roughly $17 \%$ of subjects $(3 / 19)$ saw increased post-game grip strength for the dominant arm for the over-stride condition versus $21 \%$ (4/ 19) who demonstrated greater dominant arm grip strength for the under-stride condition. Despite observed strength gains, most of the athletes in this study showed weaker dominant arm grip strength for both simulated games for over-stride and under-stride conditions.

\section{DISCUSSION}

Functional forearm fatigue of the medial elbow stabilizers increased during the condition of a simulated game outing with a shorter stride length, confirming the study's hypothesis. Linked to other work, a reduction in stride length, which can be considered a surrogate for reduced ground reaction forces sustained during pitching, can potentially increase muscular effort of the dynamic stabilizers of the medial elbow joint. ${ }^{16}$ Overall, pitchers involved in this study showed weaker non-dominant strength versus the dominant arm at baseline and did not present a shift toward greater non-dominant strength compared to the dominant arm after pitch accumulation. Thus, to monitor FFF in games, one must assess the strength of the dominant arm's flexor pronator mass as the non-dominant arm did not respond significantly to pitch accumulation.

Valgus stress testing has clearly elucidated the role of the medial elbow stabilizers offered by the flexor pronator mass. In an unloaded, loaded and loaded position with maximum grip force, isometric contraction of the flexor pronator mass saw the joint gap of the medial elbow close by $103 \mathrm{~mm}$ and reduce opening by $21 \%$ with an effect size of 1.01 , being a large effect related to isometric flexor pronator mass strength. ${ }^{24}$ Isometric strength of the flexor pronator mass is critical as moderate to high activity in the muscle group has been seen during the late cocking stage and acceleration, which is considered the critical instant in pitching with the highest kinetic properties. ${ }^{25,26}$ Wang et $\mathrm{al}^{27}$ performed a pre-fatiguing protocol to examine the effect of FFF on recruitment during pitching and found that the flexor carpi ulnaris increased neural activation by approximately $10 \%$ MVC. The fatigue protocol revealed that under fatigue, elevated recruitment of the flexor carpi ulnaris may contribute to elevated muscular effort by a principle medial elbow stabilizer while no changes were seen in elbow kinetics or ball velocity. ${ }^{27}$

The results shed insight into a clinical opportunity to optimize stride length through grip dynamometry testing. The impacts of a shortened stride, or stride length that is not properly normalized to height, can propose a potential injury risk that could stem from an acute episode in an isolated game, but also exacerbated by high repetitive loads that can impact the recovery of the medial elbow stabilizers. Although strength loss was evident, what remains unknown (and was not measured in this study) was how stride length reduction increased variability in elbow valgus torque. Within-subject variability in elbow valgus load had been modeled where the addition of fatigue to reduce load capacity of the UCL, higher load variability, and pitch repetition, collectively lowered the injury threshold and increased theoretical risk. ${ }^{28}$ Combined with the current study's results, identifying compensatory mechanisms that increase elbow valgus load variability and, in particular, altered mechanics that reduce one's ability to withstand altered loading patterns through a reduction in grip strength, should be a principle focus of study in injury prevention. Current methods used to determine fatigue in games stem from the use of radar guns. Since previous work has identified no significant changes in ball velocity during an outing, and in some cases increases in ball velocity over a sea- 
Table 2. Bilateral Strength Changes for All Subjects for Over-Stride and Under-Stride Simulated Game Conditions

\begin{tabular}{|c|c|c|c|c|c|c|c|c|c|c|c|c|}
\hline \multirow[b]{3}{*}{ SUBJECTS } & \multicolumn{6}{|c|}{ OVER-STRIDE } & \multicolumn{6}{|c|}{ UNDER-STRIDE } \\
\hline & \multicolumn{3}{|c|}{ DOMINANT ARM } & \multicolumn{3}{|c|}{ NON-DOMINANT ARM } & \multicolumn{3}{|c|}{ DOMINANT ARM } & \multicolumn{3}{|c|}{ NON-DOMINANT ARM } \\
\hline & $\begin{array}{l}\text { Pre } \\
(\mathrm{kg})\end{array}$ & $\begin{array}{l}\text { Post } \\
\text { (kg) }\end{array}$ & $\Delta$ & $\begin{array}{l}\text { Pre } \\
(\mathrm{kg})\end{array}$ & $\begin{array}{l}\text { Post } \\
\text { (kg) }\end{array}$ & $\Delta$ & $\begin{array}{l}\text { Pre } \\
(\mathrm{kg})\end{array}$ & $\begin{array}{l}\text { Post } \\
\text { (kg) }\end{array}$ & $\Delta$ & $\begin{array}{l}\text { Pre } \\
(\mathrm{kg})\end{array}$ & $\begin{array}{l}\text { Post } \\
\text { (kg) }\end{array}$ & $\Delta$ \\
\hline 1 & 44.0 & 51.8 & 7.9 & 40.7 & 50.4 & 9.6 & 39.8 & 36.8 & -3.1 & 36.9 & 33.7 & -3.2 \\
\hline 2 & 39.5 & 39.0 & -0.5 & 38.8 & 38.6 & -0.2 & 37.1 & 33.3 & -3.8 & 35.9 & 38.4 & 2.5 \\
\hline 3 & 35.4 & 36.2 & 0.9 & 38.3 & 34.7 & -3.6 & 57.3 & 54.9 & -2.4 & 55.9 & 52.8 & -3.2 \\
\hline 4 & 38.0 & 35.6 & -2.4 & 40.0 & 39.4 & -0.6 & 43.4 & 40.7 & -2.6 & 36.8 & 38.2 & 1.4 \\
\hline 5 & 44.5 & 42.1 & -2.4 & 37.9 & 41.6 & 3.7 & 40.2 & 40.9 & 0.6 & 45.6 & 44.5 & -1.0 \\
\hline 6 & 59.9 & 59.3 & -0.6 & 59.6 & 59.1 & -0.5 & 44.5 & 37.2 & -7.3 & 44.3 & 42.9 & -1.4 \\
\hline 7 & 37.3 & 37.6 & 0.3 & 40.8 & 39.3 & -1.5 & 48.1 & 51.3 & 3.2 & 40.0 & 41.8 & 1.7 \\
\hline 8 & 57.8 & 51.6 & -6.3 & 41.9 & 41.4 & -0.5 & 54.1 & 51.6 & -2.5 & 50.5 & 45.0 & -5.5 \\
\hline 9 & 54.9 & 59.7 & 4.8 & 51.9 & 59.9 & 8.0 & 43.8 & 40.5 & -3.2 & 44.5 & 44.1 & -0.5 \\
\hline 10 & 50.0 & 49.0 & -1.0 & 50.2 & 45.9 & -4.3 & 37.5 & 40.1 & 2.6 & 42.0 & 43.1 & 1.1 \\
\hline 11 & 45.0 & 43.5 & -1.5 & 44.1 & 44.7 & 0.6 & 38.0 & 34.5 & -3.5 & 36.8 & 33.8 & -3.0 \\
\hline 12 & 39.7 & 39.8 & 0.1 & 40.8 & 37.6 & -3.1 & 49.7 & 47.3 & -2.4 & 55.0 & 49.3 & -5.7 \\
\hline 13 & 38.3 & 36.9 & -1.4 & 39.2 & 38.9 & -0.4 & 44.7 & 42.4 & -2.3 & 45.1 & 40.9 & -4.2 \\
\hline 14 & 54.5 & 44.5 & -10.0 & 54.0 & 49.4 & -4.6 & 50.4 & 52.9 & 2.4 & 51.6 & 49.1 & -2.5 \\
\hline 15 & 37.5 & 35.2 & -2.3 & 35.6 & 43.9 & 8.3 & 56.7 & 47.2 & -9.5 & 50.1 & 51.9 & 1.8 \\
\hline 16 & 47.7 & 43.7 & -4.0 & 44.3 & 42.9 & -1.4 & 34.7 & 34.4 & -0.2 & 41.9 & 41.6 & -0.3 \\
\hline 17 & 32.5 & 36.9 & 4.3 & 38.9 & 38.6 & -0.2 & 38.9 & 34.7 & -4.2 & 36.3 & 38.2 & 1.9 \\
\hline 18 & 42.5 & 34.6 & -7.9 & 44.1 & 36.0 & -8.1 & 54.0 & 55.4 & 1.4 & 58.6 & 63.2 & 4.6 \\
\hline 19 & 49.4 & 47.5 & -2.0 & 43.8 & 49.9 & 6.1 & 45.9 & 45.2 & -0.7 & 45.3 & 44.6 & -0.7 \\
\hline Mean & 44.6 & 43.4 & -1.3 & 43.4 & 43.8 & 0.4 & 45.1 & 43.2 & ${ }^{*}-1.9$ & 44.9 & 44.0 & -0.8 \\
\hline Std & 8.0 & 7.9 & 4.2 & 6.3 & 7.2 & 4.7 & 7.0 & 7.4 & 3.3 & 7.1 & 7.1 & 2.8 \\
\hline
\end{tabular}

* Dominant arm grip strength was reduced by $1.9 \mathrm{~kg}$ post-game from pre-game baseline for the under-stride game condition (p =0.017). Pre; Pre-Game, Post; Post-Game.

son, perhaps radar gun velocities may not be able to detect biomechanical compensations. ${ }^{22}$ Thus, clinical strength assessments of the flexor pronator mass to assess throwing arm fatigue post-game from baseline testing may be more valuable in order to understand changes in mechanical efficiency as determined by the percentage of strength retained from the baseline measurement. As an application of the findings of this work, grip strength losses greater than 1.9 $\mathrm{kg}$ may alert coaches to examine pitching mechanics and implement subsequent musculoskeletal tests for range of motion and strength changes in proximal segments.

There are a few limitations to the investigation. First, the research involved only 19 athletes with small, but significant effects $(d=0.28)$. The small effect size calculation revealed that stride length may not have had a strong clinical influence on flexor-pronator strength loss, yet further research undertaken with greater statistical power could yield different results. The authors also cannot conclude that a decrease in grip strength is causally related to an increase in elbow valgus stress, as this study only focused on strength loss and its potential to reduce dynamic stability of the elbow. Joint mechanics were not explored in this work; therefore, FFF requires further investigation either with wearable technology or an optical tracking system to better understand how lower body mechanics influence joint loads in relation to grip strength loss. The results of this study are not generalizable to competitive game play, as stride length changes may not be as drastic, yet the model does provide an approach to inspire future work dedicated to stride length optimization, examining biomechanical responses along a variety of normalized body height spectrums. At the time of the completion of this work, the 20-second pitch clock rule had not been instituted in professional baseball; therefore, it is possible that the allocated rest times in this work could encourage additional fatigue by comparison to current conditions in minor league play. Responses seen between stride length extremes also cannot infer changes occurring from desired stride length, which is a goal of future study. A simple AB cross-over design (common in early phase studies) was utilized because the advantage is that each subject served as their own control and a smaller number of participants are required when compared to parallelgroup designs. The disadvantage of this methodological design was the inability for comparing desired stride length data with the $\pm 25 \%$ stride conditions. To enable comparison with desired stride length, more complicated crossover designs are necessary, perhaps the same desired stride length condition being administered in multiple different periods (e.g. ABAC or ABCABC designs) or to deploy a parallelgroup design $(\mathrm{ABC})$ requiring larger samples. 
Maximum capacity flat ground pitching may have involved higher exertion levels than when throwing from a pitching mound, and other notable limitations include the absence of cleats, and "in contest" competition, however these facets are consistent with other laboratory studies. $^{22,29}$ It is also important to note that related to game simulations being performed on flat ground, a small percentage of taller athletes required adjustment in throwing positions in order to target force plates. As such, given this work involved ground reaction force of the landing leg to determine stride length, only 40 pitches were available for taller pitchers to select the two fastest trials in the first inning and last inning for stride length determination. In the future, it is recommended that researchers evaluate laboratory constraints to ensure all 80 pitches are available for analysis. Similarly, effects may be different in studies that repeat the current methods by having pitchers throw from a mound without force plate adjustment. Although the results demonstrated a significant, strength loss of $1.9 \mathrm{~kg}$ (approximately 4 pounds) following simulated games with shortened stride lengths, the findings may not be considered clinically significant, where further work evaluating the presence of injury, or pain around such values is warranted.

\section{CONCLUSION}

Clinical evaluation of grip strength has the potential to identify altered lower body mechanics and may be considered as a safe and effective monitoring strategy to integrate with motion capture in determining optimal stride lengths for baseball pitchers.

\section{CONFLICTS OF INTEREST}

The authors of this work have no conflicts of interest to report.

Submitted: April 23, 2021 CDT, Accepted: August 17, 2021 CDT 


\section{REFERENCES}

1. Seiber K, Gupta R, McGarry MH, Safran MR, Lee TQ. The role of the elbow musculature, forearm rotation, and elbow flexion in elbow stability: an in vitro study. J Shoulder Elbow Surg.

2009;18(2):260-268.

2. Morrey BF, An K-N. Articular and ligamentous contributions to the stability of the elbow joint. Am J Sports Med. 1983;11(5):315-319.

3. Solomito MJ, Garibay EJ, Nissen CW. A biomechanical analysis of the association between forearm mechanics and the elbow varus moment in collegiate baseball pitchers. Am J Sports Med. 2018;46(1):52-57.

4. Buffi JH, Werner K, Kepple T, Murray WM. Computing muscle, ligament, and osseous contributions to the elbow varus moment during baseball pitching. Ann Biomed Eng. 2015;43(2):404-415.

5. Sonne MWL, Keir PJ. Major League Baseball paceof-play rules and their influence on predicted muscle fatigue during simulated baseball games. J Sports Sci. 2016;34(21):2054-2062.

6. Olsen SJ, Fleisig GS, Dun S, Loftice J, Andrews JR. Risk factors for shoulder and elbow injuries in adolescent baseball pitchers. Am J Sports Med. 2006;34(6):905-912. doi:10.1177/0363546505284188

7. Mullaney MJ, McHugh MP, Donofrio TM, Nicholas SJ. Upper and lower extremity muscle fatigue after a baseball pitching performance. Am J Sports Med. 2005;33(1):108-113.

8. Yanagisawa O, Taniguchi H. Changes in lower extremity function and pitching performance with increasing numbers of pitches in baseball pitchers. $J$ Exerc Rehabil. 2018;14(3):430.

9. Grantham WJ, Byram IR, Meadows MC, Ahmad CS. The impact of fatigue on the kinematics of collegiate baseball pitchers. Orthop J Sport Med. 2014;2(6):232596711453703. doi:10.1177/2325967114 $\underline{537032}$

10. Escamilla RF, Barrentine SW, Fleisig GS, et al. Pitching biomechanics as a pitcher approaches muscular fatigue during a simulated baseball game. Am J Sports Med. 2007;35(1):23-33. doi:10.1177/03635 46506293025
11. Okoroha KR, Meldau JE, Lizzio VA, et al. Effect of fatigue on medial elbow torque in baseball pitchers: a simulated game analysis. Am J Sports Med. 2018;46(10):2509-2513.

12. Grantham WJ, Byram IR, Meadows MC, Ahmad $\mathrm{CS}$. The impact of fatigue on the kinematics of collegiate baseball pitchers. Orthop J Sport Med. 2014;2(6):2325967114537032.

13. Crotin RL, Kozlowski K, Horvath P, Ramsey DK. Altered stride length in response to increasing exertion among baseball pitchers. Med Sci Sports Exerc. 2014;46(3):565-571. doi:10.1249/MSS.0b013e3 $\underline{182 \mathrm{a} 79 \mathrm{~cd} 9}$

14. Ramsey DK, Crotin RL. Effect of stride length on overarm throwing delivery: Part II: An angular momentum response. Hum Mov Sci. 2016;46:30-38. d oi:10.1016/j.humov.2015.11.021

15. Crotin RL, Bhan S, Ramsey DK. An inferential investigation into how stride length influences temporal parameters within the baseball pitching delivery. Hum Mov Sci. 2015;41:127-135. doi:10.1016/ j.humov.2015.03.005

16. Ramsey DK, Crotin RL. Stride length: the impact on propulsion and bracing ground reaction force in overhand throwing. Sport Biomech. 2019;18:553-570.

17. Kibler WB. The role of the scapula in athletic shoulder function. Am J Sports Med. 1998;26(2):325-337.

18. Ramsey DK, Crotin RL, White S. Effect of stride length on overarm throwing delivery: A linear momentum response. Hum Mov Sci. 2014;38:185-196. doi:10.1016/j.humov.2014.08.012

19. Kobesova A, Dzvonik J, Kolar P, Sardina A, Andel R. Effects of shoulder girdle dynamic stabilization exercise on hand muscle strength. Isokinet Exerc Sci. 2015;23(1):21-32.

20. Fleisig GS, Kingsley DS, Loftice JW, et al. Kinetic comparison among the fastball, curveball, change-up, and slider in collegiate baseball pitchers. Am J Sports Med. 2006;34(3):423-430.

21. Fleisig GS, Barrentine SW, Zheng N, Escamilla RF, Andrews JR. Kinematic and kinetic comparison of baseball pitching among various levels of development. J Biomech. 1999;32(12):1371-1375. 
22. Crotin R, Bhan S, Karakolis T, Ramsey D. Fastball velocity trends in short-season minor league baseball. J Strength Cond Res. 2013;27(8):2206-2212. doi:10.151 9/JSC.0b013e31827e1509

23. Crotin RL, Ramsey DK. Stride length: A reactive response to prolonged exertion potentially effecting ball velocity among baseball pitchers. Int J Perform Anal Sport. 2015;15(1). doi:10.1080/24748668.2015.11 $\underline{868791}$

24. Pexa BS, Ryan ED, Myers JB. Medial elbow joint space increases with valgus stress and decreases when cued to perform a maximal grip contraction. Am J Sports Med. 2018;46(5):1114-1119.

25. DiGiovine NM, Jobe FW, Pink M, Perry J. An electromyographic analysis of the upper extremity in pitching. J Shoulder Elb Surg. 1992;1(1):15-25.
26. Fleisig GS, Andrews JR, Dillman CJ, Escamilla RF. Kinetics of baseball pitching with implications about injury mechanisms. Am J Sports Med. 1995;23(2):233-239.

27. Wang LH, Lo KC, Jou IM, Kuo LC, Tai TW, Su FC. The effects of forearm fatigue on baseball fastball pitching, with implications about elbow injury. $J$ Sports Sci. 2016;34(12):1182-1189. doi:10.1080/02640 $\underline{414.2015 .1101481}$

28. Trigt van B, Leenen TAJR, Hoozemans MMJM, Helm FFCT, Veeger DHEJ. Are UCL Injuries a Matter of Bad Luck? The Role of Variability and Fatigue Quantified. Multidisciplinary Digital Publishing Institute Proceedings. 2020;49:107.

29. MacWilliams BA, Choi T, Perezous MK, Chao EYS, McFarland EG. Characteristic ground-reaction forces in baseball pitching. Am J Sports Med. 1998;26(1):66-71. 